\title{
Association Between Before-bedtime Passive Body Heating and Nocturia During the Cold Season Among Older Adults
}

Yoshiaki Tai $^{1}$, Kenji Obayashi ${ }^{1}$, Kazuki Okumura ${ }^{1,2}$, Yuki Yamagami ${ }^{1}$, Hiromitsu Negoro $^{3}$, Norio Kurumatani ${ }^{1}$, and Keigo Saeki ${ }^{1}$

${ }^{1}$ Department of Epidemiology, Nara Medical University School of Medicine, Nara, Japan

${ }^{2}$ Department of Psychiatry, Nara Medical University School of Medicine, Nara, Japan

${ }^{3}$ Department of Urology, University of Tsukuba, Ibaraki, Japan

Received November 12, 2021; accepted January 26, 2022; released online February 19, 2022

\begin{abstract}
Background: Cold exposure induces lower urinary tract symptoms, including nocturia. Cold-induced detrusor overactivity can be alleviated by increasing skin temperature in rats. However, no study has shown an association between passive heating via hot-water bathing and nocturia among humans.

Methods: We included 1,051 Japanese community-dwelling older adults (mean age: 71.7 years) in this cross-sectional study from 2010 to 2014. The number of nocturnal voids was recorded in a self-administered urination diary. Nocturia was defined as $\geq 2$ nocturnal voids. We evaluated bathing conditions in the participants' houses.

Results: Hot-water bathing $(n=888)$ was associated with a lower prevalence of nocturia than no bathing $(n=163)$, independent of potential confounders, including age, sex, obesity, income, physical activity, diabetes, medication (diuretics, nondiuretic antihypertensives, and hypnotics), depressive symptoms, indoor/outdoor temperature, and day length (odds ratio [OR] 0.68; 95\% confidence interval $[\mathrm{CI}], 0.48-0.97 ; P=0.035)$. Compared with the quartile group with the longest bath-to-bed interval (range: 161-576 $\mathrm{min}$ ), the second and third quartile groups (range: 61-100 and 101-160 min, respectively) were associated with a lower prevalence of nocturia, after adjusting for water temperature and bathing duration besides the same covariates (OR 0.60; 95\% CI, 0.38-0.96; $P=0.031$ and OR 0.59; 95\% CI, 0.37-0.94; $P=0.025$, respectively).

Conclusion: Hot-water bathing, particularly with a bath-to-bed interval of 61-160 min, was significantly associated with a lower prevalence of nocturia among older adults.
\end{abstract}

Key words: nocturia; lower urinary tract symptoms; cold temperature; baths; passive body heating; hyperthermia

Copyright $\odot 2022$ Yoshiaki Tai et al. This is an open access article distributed under the terms of Creative Commons Attribution License, which permits unrestricted use, distribution, and reproduction in any medium, provided the original author and source are credited.

\section{INTRODUCTION}

Nocturia, defined as waking to pass urine during the main sleep period, ${ }^{1}$ is an unpleasant symptom, and $\geq 2$ nocturia episodes are clinically significant. ${ }^{2} \mathrm{Up}$ to $59.3 \%$ of adults aged $\geq 70$ years experience $\geq 2$ nocturia episodes. ${ }^{3}$ The condition is associated with various morbidities, such as obesity, diabetes, hypertension, cardiac disease, and depression; these morbidities may contribute to a higher risk of mortality. ${ }^{4-6}$

Cold exposure results in lower urinary tract symptoms (LUTS). Previously, low ambient temperature-induced detrusor overactivity in rats was found to be correlated with decreased skin temperature. $^{7}$ Epidemiological studies have reported that the prevalence of nocturia was high in winter and in indoor environments with low temperatures. ${ }^{8,9}$

Passive body heating (PBH) can alleviate cold stress, ${ }^{10}$ and is associated with the causative factors of nocturia. Previous studies have shown that hot-water bathing with optimal timing is associated with a higher skin temperature at bedtime, higher sleep efficiency, shorter sleep onset latency, and lower night-time blood pressure. ${ }^{11-13}$ Moreover, high-frequency hot-water bathing was correlated with a lower B-type natriuretic peptide level. ${ }^{14} \mathrm{~A}$ randomized crossover study involving seven female inpatients aged $\geq 65$ years found no significant effect of foot bathing on night-time urinary frequency, but the sample size may have been insufficient. ${ }^{15}$ In a previous randomized controlled trial (RCT), an eight-item behavioral therapy, including taking a bath, was associated with a reduced number of nocturnal voids, although the achievement rate of taking a bath was only $41 \%{ }^{16}$

Although hot-water bathing before bedtime is a prevailing practice in Japan, particularly among older adults, and is related to the causative factors of nocturia, to our knowledge, no study has reported an association between PBH with hot-water bathing and nocturia. Hence, the present study was conducted to investigate the association between $\mathrm{PBH}$ via hot-water bathing and nocturia among community-dwelling older adults. The association between bathing conditions and nocturia was also evaluated. 


\section{METHODS}

\section{Participants and study protocol}

This cross-sectional analysis was performed using baseline data from the HEIJO-KYO cohort. ${ }^{17}$ This cohort study included 1,127 community-dwelling older individuals aged $\geq 60$ years who were voluntarily assembled with the cooperation of local associations of older adults. The baseline survey was conducted between September 2010 and April 2014. The 1,122 participants were assessed during the cold season (from October to April). We excluded 16 and 55 participants who did not complete a selfadministered urination diary and the measurements of bathing conditions, respectively. Therefore, a total of 1,051 participants were assessed. The study protocol has been described in our previous study. ${ }^{17}$ In summary, we visited each participant's residence and assessed the indoor environment and their behavior, including bathing, for 48 consecutive hours from the noon of the first day. Nocturnal urination was evaluated on the second night. After receiving sufficient explanation regarding the study, all participants gave their written informed consent. This study was conducted in accordance with the principles of the Declaration of Helsinki and approved by the ethics committee of Nara Medical University (no. 301).

\section{Night-time frequency and urine volume}

The number of nocturnal voids except for the last void before bedtime and the first void after rising time (night-time frequency) was recorded for one night in the self-administered urination diary. Nocturia was defined as $\geq 2$ nocturnal voids. Each participant collected urine samples in a bottle during night-time, which included the first morning void (nocturnal urine volume). After retrieving the bottle, we measured and recorded the total volume. The nocturnal urine production rate $(\mathrm{mL} /$ hour) was calculated as nocturnal urine volume divided by time spent in bed. Single voided urine volume was calculated as nocturnal urine volume divided by the number of nocturnal voids including the first morning void.

\section{Bathing conditions and indoor temperature}

The presence or absence of hot-water bathing, which was defined as taking a bathtub bath (excluding taking a shower), was recorded in a self-administered bathing diary. The start and end time of bathing, time in the bathtub (bathing duration), bedtime, and rising time were logged in the diary. The interval from the start time of bathing to bedtime (bath-to-bed interval) was calculated as bedtime minus the start time of bathing. The bathtub water temperature was measured using a logger (Thermochron iButton; Maxim Integrated, Dallas, TX, USA) at 1-minute intervals. The bathroom, dressing room, and living room temperatures were measured $60 \mathrm{~cm}$ above the floor at 10-minute intervals using the same logger, which we placed in each participants' home during the consecutive 48-hour session. We calculated the mean temperature of the bathtub water (hot-water temperature), bathroom, and dressing room while the participants were in the bathroom, according to the record. Because of technical errors, we excluded some data on hot-water temperature using the same methods previously reported. ${ }^{12}$ Daytime indoor temperature was defined as the mean living room temperature between rising time and bedtime of the second day.

\section{Other measurements}

Body mass index (BMI) was calculated as body weight $(\mathrm{kg})$ divided by height $\left(\mathrm{m}^{2}\right)$. The presence of obesity was defined as BMI $\geq 25 \mathrm{~kg} / \mathrm{m}^{2}$. Current smoking and drinking habits, household income, medication use, and medical history were reported using a self-administered questionnaire and were confirmed via a medical interview. Diabetes mellitus was determined based on medication use, medical history, or glycated hemoglobin $\geq 6.5 \%$. Estimated glomerular filtration rate (eGFR) was calculated using the estimating equation for the Japanese population. ${ }^{18}$ Physical activity level was assessed using the Japanese version of the International Physical Activity Questionnaire. ${ }^{19,20}$ Participants with depressive symptoms were defined as participants with a score higher than five on the 15-item Geriatric Depression Scale (GDS). ${ }^{21}$ Day length (between sunrise and sunset) in Nara on the measurement day was extracted using data from the National Astronomical Observatory of Japan. Outdoor temperature at 10minute intervals was estimated using data from the local meteorological office in Nara (latitude, $34^{\circ} \mathrm{N}$ ). Daytime outdoor temperature was defined as the mean outdoor temperature between rising time and bedtime.

\section{Statistical analysis}

Normally distributed variables were presented as mean and standard deviation (SD), and non-normally distributed variables as median and interquartile ranges. Means, medians, and proportions were compared using the unpaired $t$-test, MannWhitney U test, and $\chi^{2}$ test, respectively. Single voided urine volume was determined after natural log-transformation because of its non-normal distribution. Trends in the association between night-time frequency and bathing/sleep variables were evaluated using the linear regression model.

Logistic regression models were used to calculate the odds ratio (OR) of nocturia between the bathing and no bathing groups. The OR was adjusted for potential confounders, including basic variables (age [years], sex, obesity [BMI $\geq 25 \mathrm{~kg} / \mathrm{m}^{2}$ ], current smoking status, alcohol consumption [ $\geq 30 \mathrm{vs}<30 \mathrm{~g} /$ day], household income [ $\geq 4$ vs $<4$ million Japanese yen]), clinical variables (use of diuretics, nondiuretic antihypertensives, hypnotics, depressive symptoms [15-item GDS $\geq 6$ ], diabetes mellitus, eGFR $\left[\geq 60\right.$ vs $<60 \mathrm{~mL} / \mathrm{min} / 1.73 \mathrm{~m}^{2}$ ], physical activity [per quartile change]), and environmental variables (daytime indoor temperature $\left[{ }^{\circ} \mathrm{C}\right]$, daytime outdoor temperatures $\left[{ }^{\circ} \mathrm{C}\right]$, daylength [per quartile change]). The difference in log-transformed single voided urine volume between the bathing and no bathing group was calculated using the linear regression model adjusted for basic, clinical, and environmental variables.

To evaluate the associations of bath-to-bed interval, bathing duration, and hot-water temperature with nocturia, we performed a subgroup analysis of participants who bathed. They were divided into four groups according to the quartile values of the bath-to-bed interval due to its non-normal distribution. The ORs of nocturia between the Q1, Q2, and Q3 groups and the Q4 group of bath-to-bed interval were calculated because the Q4 group may have had the lowest heat load remaining at bedtime. The ORs were adjusted for bathing variables, bedtime (clock time), and time in bed (hours), in addition to all covariates used in the analysis between the bathing and no bathing groups. The Poisson regression models with generalized estimating equations were used to evaluate the association of the three bathing variables with night-time frequency by calculating the ratios of night-time 
Tai Y, et al.

Table 1. Basic, clinical, and environmental characteristics of participants $(n=1,051)$

\begin{tabular}{|c|c|c|c|}
\hline & \multicolumn{2}{|c|}{ Hot-water bathing } & \multirow{2}{*}{$P$} \\
\hline & without $(n=163)$ & with $(n=888)$ & \\
\hline \multicolumn{4}{|l|}{ Basic variables } \\
\hline Age, years, mean (SD) & $74.6(7.3)$ & $71.2(6.9)$ & $<0.001$ \\
\hline Sex, female, $n(\%)$ & $93(57.1)$ & $469(52.8)$ & 0.318 \\
\hline Body mass index, $\mathrm{kg} / \mathrm{m}^{2}$, mean (SD) & $22.6(3.1)$ & $23.2(3.0)$ & 0.029 \\
\hline Current smoker, $n(\%)$ & $9(5.5)$ & $43(4.8)$ & 0.713 \\
\hline Alcohol consumption, $\geq 30 \mathrm{~g} /$ day, $n(\%)$ & $16(9.8)$ & $135(15.2)$ & 0.072 \\
\hline Household income, $\geq 4$ million JPY/year, $n(\%)$ & $48(31.4)$ & $379(46.2)$ & 0.001 \\
\hline \multicolumn{4}{|l|}{ Clinical variables } \\
\hline Diabetes mellitus, $n(\%)$ & $22(13.6)$ & $98(11.2)$ & 0.387 \\
\hline $\mathrm{eGFR}<60 \mathrm{~mL} / \mathrm{min} / 1.73 \mathrm{~m}^{2}, n(\%)$ & $31(19.0)$ & $157(17.9)$ & 0.729 \\
\hline Diuretics use, $n(\%)$ & $8(4.9)$ & $52(5.9)$ & 0.632 \\
\hline Nondiuretic antihypertensives use, $n(\%)$ & $63(38.7)$ & $343(38.6)$ & 0.995 \\
\hline Sleep medication use, $n(\%)$ & $18(11.0)$ & $84(9.5)$ & 0.536 \\
\hline Depressive symptoms ${ }^{\mathrm{a}}, n(\%)$ & $31(19.0)$ & $125(14.1)$ & 0.119 \\
\hline Physical activity, MET-minutes/week, median (IQR) & $1,188(330-2,613)$ & $1,371(495-2,772)$ & 0.190 \\
\hline \multicolumn{4}{|l|}{ Environmental variables } \\
\hline Daytime outdoor temperature, mean $(\mathrm{SD}),{ }^{\circ} \mathrm{C}$ & $8.7(4.3)$ & $8.5(5.0)$ & 0.653 \\
\hline Daytime indoor temperature, mean $(\mathrm{SD}),{ }^{\circ} \mathrm{C}$ & $15.3(4.3)$ & $15.8(3.8)$ & 0.144 \\
\hline Day length, minutes, median (IQR) & $641(613-674)$ & $656(623-684)$ & 0.023 \\
\hline \multicolumn{4}{|l|}{ Variables on nocturnal urination } \\
\hline Nocturnal urine volume, mL, mean (SD) & $563.3(269.6)$ & $570.7(254.3)$ & 0.745 \\
\hline Nocturnal urine production rate, $\mathrm{mL} /$ hour, mean $(\mathrm{SD})$ & $66.8(33.8)$ & $70.7(31.5)$ & 0.152 \\
\hline Single voided urine volume, $\mathrm{mL}$, median $(\mathrm{IQR})^{\mathrm{b}}$ & $250(173-330)$ & $282(205-380)$ & 0.004 \\
\hline Night-time frequency $\geq 2$ voids, $n(\%)$ & $66(40.5)$ & $242(27.3)$ & 0.001 \\
\hline
\end{tabular}

eGFR, estimated glomerular filtration rate; JPY, Japanese yen; SD, standard deviation.

$P$-values were calculated using the unpaired $t$-test and $\chi^{2}$ test.

${ }^{\mathrm{a}} 15$-item Geriatric Depression Scale $\geq 6$.

${ }^{\mathrm{b}}$ Tested after natural log-transformation

frequency (ratios of the Q1/Q4, Q2/Q4, and Q3/Q4 groups of the bath-to-bed interval). The ratios were adjusted for the same covariates used in the logistic regression models in the subgroup analysis.

All analyses were performed using SPSS for Windows (version 26.0; IBM SPSS Inc., Chicago, IL, USA). All $P$-values were two-sided, and a $P$-value of $<0.05$ was considered statistically significant.

\section{RESULTS}

\section{Baseline demographic and clinical characteristics}

Of 1,051 participants (mean age: 71.7; SD, 7.1 years), 562 (53.5\%) were women, $888(84.5 \%)$ bathed, and 308 (29.3\%) experienced nocturia. Significant differences were found in age, day length, and single voided urine volume; proportions of obesity and household income ( $>4$ million Japanese yen); and prevalence of nocturia between the bathing and no bathing groups (Table 1). By contrast, there were no significant differences in the nocturnal urine volume or nocturnal urine production rate between the two groups.

\section{Hot-water bathing and nocturia}

Compared with the no bathing group, the bathing group was significantly associated with a lower prevalence of nocturia (Table 2). The OR of nocturia between the bathing and no bathing groups was 0.68 (95\% confidence interval [CI], 0.48$0.97 ; P=0.035$ ) after adjusting for basic variables (age, sex, obesity, current smoking, alcohol consumption, and household income), clinical variables (diabetes mellitus, eGFR, medication
Table 2. Odds ratio of nocturia associated with hot-water bathing

\begin{tabular}{lccc}
\hline & \multicolumn{2}{c}{ Hot-water bathing } & \multirow{2}{*}{$P$ value } \\
\cline { 2 - 3 } & without $(n=163)$ & with $(n=888)$ & \\
\hline Unadjusted OR (95\% CI) & 1.00 (Ref.) & $0.55(0.39-0.78)$ & 0.001 \\
& & & \\
Adjusted OR (95\% CI) & & & \\
Model 1 & 1.00 (Ref.) & $0.67(0.47-0.94)$ & 0.019 \\
Model 2 $^{\mathrm{b}}$ & 1.00 (Ref.) & $0.67(0.47-0.95)$ & 0.025 \\
Model 3 $^{\mathrm{c}}$ & 1.00 (Ref.) & $0.68(0.48-0.97)$ & 0.035 \\
\hline
\end{tabular}

$\mathrm{CI}$, confidence interval; OR, odds ratio; Ref., reference.

ORs of nocturia were calculated using the logistic regression model.

${ }^{a}$ Adjusted for age, sex, and body mass index $\left(\geq 25 \mathrm{~kg} / \mathrm{m}^{2}\right)$.

${ }^{\mathrm{b}}$ Adjusted for current smoking, alcohol consumption ( $\geq 30 \mathrm{~g} /$ day), household income ( $\geq 4$ million Japanese yen), diabetes mellitus, estimated glomerular filtration rate $\left(<60 \mathrm{~mL} / \mathrm{min} / 1.73 \mathrm{~m}^{2}\right)$, medication use (diuretics, nondiuretic antihypertensives, hypnotics), depressive symptoms (15-item Geriatric Depression scale $\geq 6$ ), physical activity (per quartile change), in addition to Model 1.

${ }^{\mathrm{c}}$ Adjusted for daytime outdoor and indoor temperatures, and day length (per quartile change), in addition to Model 2.

use [diuretics, nondiuretic antihypertensives, and hypnotics], depressive symptoms, and physical activity [per quartile change]), and environmental variables (daytime indoor and outdoor temperatures, and day length [per quartile change]) (model 3 in Table 2). The single voided urine volume of the bathing group was higher than that of no bathing group after adjusting for basic, clinical, and environmental variables (mean difference: $0.083 \log -\mathrm{mL} ; 95 \% \mathrm{CI}, 0.004-0.163 ; P=0.040$ ). 


\section{Bathing conditions and nocturia}

A higher night-time urinary frequency was significantly associated with later bathing time, longer bath-to-bed interval, later bedtime, and longer time in bed (Table 3). The Q2 (61-100 minute) and Q3 (101-160 minute) groups of bath-to-bed interval were significantly associated with a lower prevalence of nocturia than the Q4 (161-576 minute) group (Table 4). After adjusting for basic, clinical, and environmental variables, in addition to bathing duration, hot-water temperature, bedtime, and time in bed, the associations remained significant (adjusted OR in Table 4). By contrast, bathing duration and hot-water temperature were not significantly associated with the prevalence of nocturia in the adjusted model.

In the Poisson regression analysis, night-time frequency in the Q2 and Q3 groups of bath-to-bed interval were significantly lower than those in the Q4 group (eTable 1). The ratios of nighttime frequency (the ratios of the Q1/Q4, Q2/Q4 and Q3/Q4 group of bath-to-bed interval) were 0.90 (95\% CI, 0.78-1.04;
$P=0.163), 0.82$ (95\% CI, 0.71-0.96; $P=0.013)$, and $0.86(95 \%$ CI, 0.74-0.99; $P=0.048)$, respectively, after adjusting for basic, clinical, environmental variables, in addition to bathing duration, hot-water temperature, bedtime and time in bed (adjusted nocturnal voids ratio in eTable 1). By contrast, there was no significant association of bathing duration and hot-water temperature with night-time frequency in the adjusted model.

\section{DISCUSSION}

In this study, PBH via hot-water bathing was associated with a lower prevalence of nocturia in the cold environment, independent of potential confounders, such as age, sex, obesity, medication use, physical activity, and indoor temperature. Hotwater bathing with a bath-to-bed interval of 61-160 minutes was significantly associated with a lower prevalence of nocturia and night-time frequency than a bath-to-bed interval of $\geq 161$ minutes. Furthermore, single voided urine volume, a surrogate for

Table 3. Bathing and sleep parameters stratified by night-time frequency

\begin{tabular}{|c|c|c|c|c|c|c|}
\hline & \multirow{2}{*}{ All } & \multicolumn{4}{|c|}{ Night-time frequency (voids/night) } & \multirow{2}{*}{$P$ trend } \\
\hline & & 0 & 1 & 2 & $\geq 3$ & \\
\hline No. of participants & 888 & 250 & 396 & 177 & 65 & \\
\hline \multicolumn{7}{|l|}{ Bathing parameters, mean (SD) } \\
\hline Start time of bathing & $20: 34(1: 48)$ & $21: 07(1: 45)$ & $20: 36(1: 38)$ & $19: 58(1: 59)$ & $19: 46(1: 34)$ & $<0.001$ \\
\hline End time of bathing & $21: 02(1: 48)$ & $21: 35(1: 45)$ & $21: 04(1: 45)$ & $20: 26(1: 59)$ & $20: 13(1: 36)$ & $<0.001$ \\
\hline Bath-to-bed interval, $\min ^{\mathrm{a}}$ & $100.0(60.0-160.0)$ & $93.5(60-150)$ & $102(61-158)$ & $108(60-177.5)$ & $115(65-195)$ & 0.044 \\
\hline Bathing duration, min & $13.0(6.9)$ & $12.9(7.5)$ & $12.6(6.2)$ & $13.6(6.3)$ & $14.1(9.5)$ & 0.109 \\
\hline Hot water temperature, ${ }^{\circ} \mathrm{C}$ & $40.73(1.36)$ & $40.72(1.36)$ & $40.72(1.39)$ & $40.73(1.30)$ & $40.80(1.35)$ & 0.720 \\
\hline Bath room temperature, ${ }^{\circ} \mathrm{C}$ & $16.4(4.3)$ & $16.7(4.5)$ & $16.4(4.1)$ & $15.8(4.4)$ & $16.3(4.6)$ & 0.126 \\
\hline Dressing room temperature, ${ }^{\circ} \mathrm{C}$ & $13.4(4.1)$ & $13.5(4.0)$ & $13.5(4.0)$ & $13.1(4.3)$ & $13.6(4.3)$ & 0.622 \\
\hline \multicolumn{7}{|l|}{ Sleep parameters, mean (SD) } \\
\hline Bedtime & $22: 37(1: 12)$ & $23: 04(1: 12)$ & $22: 37(1: 07)$ & $22: 11(1: 11)$ & $21: 55(1: 05)$ & $<0.001$ \\
\hline Rising time & $6: 47(0: 57)$ & $6: 38(0: 59)$ & $6: 48(0: 55)$ & $6: 50(0: 59)$ & 7:01 (0:50) & $<0.001$ \\
\hline Time in bed, min & $490.9(80.5)$ & $455.0(80.5)$ & $491.6(72.7)$ & $519.7(73.7)$ & $546.8(80.3)$ & $<0.001$ \\
\hline
\end{tabular}

Bath-to-bed interval, interval from start time of bathing to bedtime; SD, standard deviation.

$P$-values for trend were calculated using the linear regression model.

${ }^{a}$ Expressed as median (interquartile ranges) and tested after natural log transformation.

Table 4. Odds ratios for nocturia according to changes in bathing and sleep variables $(n=888)$

\begin{tabular}{|c|c|c|c|c|}
\hline & $\begin{array}{l}\text { Unadjusted OR } \\
(95 \% \mathrm{CI})\end{array}$ & $P$ value & $\begin{array}{l}\text { Adjusted } \mathrm{OR}^{\mathrm{a}} \\
\quad(95 \% \mathrm{CI})\end{array}$ & $P$ value \\
\hline \multicolumn{5}{|l|}{ Bathing parameters } \\
\hline \multicolumn{5}{|l|}{ Bath-to-bed interval } \\
\hline Q1 [10-60 min] vs Q4 & $0.77(0.51-1.15)$ & 0.202 & $0.73(0.47-1.15)$ & 0.178 \\
\hline $\mathrm{Q} 2[61-100 \mathrm{~min}]$ vs $\mathrm{Q} 4$ & $0.62(0.41-0.94)$ & 0.026 & $0.60(0.38-0.96)$ & 0.031 \\
\hline Q3 [101-160 min] vs Q4 & $0.58(0.38-0.89)$ & 0.012 & $0.59(0.37-0.94)$ & 0.025 \\
\hline Q4 [161-576 min] & 1.00 (Ref.) & - & 1.00 (Ref.) & - \\
\hline Bathing duration (per $1 \mathrm{~min}$ ) & $1.02(1.00-1.04)$ & 0.047 & $1.01(0.99-1.04)$ & 0.320 \\
\hline Hot water temperature (per $1^{\circ} \mathrm{C}$ ) & $1.02(0.92-1.14)$ & 0.681 & $0.97(0.86-1.09)$ & 0.607 \\
\hline \multicolumn{5}{|l|}{ Sleep parameters } \\
\hline Bedtime (per 1 hour later) & $0.62(0.55-0.70)$ & $<0.001$ & $0.81(0.67-0.99)$ & 0.043 \\
\hline Time in bed (per 1 hour) & $1.62(1.46-1.80)$ & $<0.001$ & $1.24(1.03-1.49)$ & 0.024 \\
\hline
\end{tabular}

Bath-to-bed interval, interval from start time of bathing to bedtime; CI, confidence interval; OR, odds ratio; Ref. reference.

${ }^{a}$ Adjusted for age, sex, obesity (body mass index $\geq 25 \mathrm{~kg} / \mathrm{m}^{2}$ ), current smoking, alcohol consumption ( $\geq 30 \mathrm{~g} /$ day), household income ( $\geq 4$ million Japanese yen), diabetes mellitus, estimated glomerular filtration rate $\left(<60 \mathrm{~mL} / \mathrm{min} / 1.73 \mathrm{~m}^{2}\right)$, medication use (diuretics, nondiuretic antihypertensives, hypnotics), depressive symptoms (15-item Geriatric Depression scale $\geq 6$ ), physical activity (per quartile change), outdoor and indoor temperatures, day length (per quartile change), and all variables shown in this Table.

ORs of nocturia were calculated using the logistic regression model, which expresses the ORs to the reference group or the ORs per 1.0 unit increase in parameters. 
functional bladder capacity, was significantly higher in the bathing group. However, nocturnal urine volume and production rate were not significantly different between the two groups. To our knowledge, this is the first study that evaluated an association between $\mathrm{PBH}$ via hot-water bathing and nocturia.

Although the mechanisms underlying the association between $\mathrm{PBH}$ and nocturia remain uncertain, previous studies showed that improvement in cold-induced lower urinary tract symptoms (LUTS) with medication and exercise was associated with high skin temperature and low cold sensitivity. ${ }^{22,23}$ A galenical solution extracted from traditional herbal medicine increased skin temperature and reduced cold-induced detrusor overactivity in rats. ${ }^{22}$ Administration of the solution reduced the expression of transient receptor potential cation channel subfamily melastin member 8 , an ion channel expressed in cold-sensing neurons, ${ }^{23}$ in the skin tissues of rats. An exercise intervention conducted in the cold season increased skin temperature and reduced LUTS in individuals with cold sensitivity. ${ }^{22}$ Our previous study showed that hot-water bathing with a bath-to-bed interval of 61-180 minutes was associated with a high skin temperature at bedtime. ${ }^{11}$ The bathto-bed interval was similar to those of the Q2 and Q3 groups in the present study. In the present study, the single voided urine volume was larger in the bathing group, suggesting that the before-bedtime $\mathrm{PBH}$ is associated with a larger functional bladder capacity.

Possible explanations for the association of $\mathrm{PBH}$ with nocturia include decreased nocturnal urinary volume, improvement in sleep quality, and circadian rhythm modification. Previous studies have showed that hot-water bathing with optimal timing was associated with lower nighttime blood pressure, shorter sleep onset latency, and better sleep efficiency. ${ }^{11-13}$ An interventional study in patients with insomnia showed ramelteon alleviated insomnia, concurrent with a decrease in the number of nocturnal voids and an increase in nighttime bladder capacity, ${ }^{24}$ suggesting interactions between sleep quality and nocturia. Moreover, previous RCTs revealed that repeated $\mathrm{PBH}$ via other forms improved chronic heart failure and depression. ${ }^{25,26}$ However, there was no significant difference in nocturnal urine volume or production rate between the bathing and no bathing groups in this study. One plausible explanation for this observation is that a change in the body weight after a single session of hot-water bathing, which ranged 71-130g depending on the manner of hot-water bathing, ${ }^{27}$ could be compensated by fluid intake after bathing because this study was non-interventional. Changes in body temperature caused by environmental temperature might modify the circadian rhythm of peripheral organs, including the kidney and bladder, ${ }^{28}$ because circadian oscillations of peripheral tissues are sensitive to temperature changes within the physiological temperature range $\left(36.0-38.5^{\circ} \mathrm{C}\right) .^{29}$

Our results implied that the estimated reduction in night-time frequency in correlation with $\mathrm{PBH}$ might not be lower than that induced by medications for benign prostatic hyperplasia $(\mathrm{BPH})$ and overactive bladder $(\mathrm{OAB})$. Based on our findings regarding the night-time frequency ratio $\mathrm{Q} 2 / \mathrm{Q} 4$ group of bath-to-bed interval in the adjusted model $(0.82 ; 95 \% \mathrm{CI}, 0.70-0.96)$, the hypothetical reductions of night-time frequency in the Q2 group were $0.36(2-0.82 \times 2), 0.54(3-0.82 \times 3)$, and 0.72 $(4-0.82 \times 4)$ voids, where night-time frequency were 2,3 , and 4 voids in the Q4 group, respectively. In previous RCTs of patients with $\mathrm{BPH}$, the night-time frequency among patients treated with doxazosin and dutasteride were reduced by 0.16 and $<0.30$ voids compared with placebo, respectively. ${ }^{30,31}$ Similarly, the night- time frequency among patients with $\mathrm{OAB}$ treated with trospium chloride, which is the most effective antimuscarinic for nocturia, was reduced by 0.24 voids compared with placebo. ${ }^{32}$

The strengths of this study include the use of real-world data obtained via the detailed measurements of the indoor environment and inclusion of a large sample, which enabled multivariable analysis with clinical and environmental variables.

This study has several limitations. First, the participants were not randomly selected, which could lead to selection bias. However, some results, including BMI and eGFR, were similar to nationwide data. ${ }^{33}$ Second, residual confounding was possible because we did not confirm whether BPH, OAB, peripheral edema, and sleep apnea were present and did not measure fluid intake and 24-hour urine volume. Third, night-time frequency was examined only for one night. The measurements may not be representative data. However, we reported the moderate reproducibility of night-time frequency in 189 participants who underwent additional assessments of night-time frequency $(\kappa$ coefficient $=0.55) .{ }^{34}$ Fourth, core body temperatures were not measured. Therefore, further studies should be performed to assess heat load, heat loss, and temperature rhythm.

$\mathrm{PBH}$ with hot-water bathing was associated with a lower prevalence of nocturia among community-dwelling older adults. A bath-to-bed interval of 61-160 minutes was significantly associated with a low prevalence of nocturia and low night-time frequency. These findings can provide a framework for future interventional studies examining the effect of $\mathrm{PBH}$ on nocturia.

\section{ACKNOWLEDGMENTS}

The authors thank Naomi Takenaka, Sachiko Sogahara, Keiko Nakajima, and Megumi Natsuaki for their valuable assistance with data collection and analyses.

Data availability: Data cannot be shared for privacy or ethical reasons.

Funding sources: This work was supported by research funding as follows.

The Department of Indoor Environmental Medicine, Nara Medical University

JSPS KAKENHI (grant numbers: 24790774, 22790567, 25860447, 25461393, 15H04776, 15H04777, 16K09478, 18H06396, 18K15899, 19K21475, 19H03903, 20K10476, 21K17310)

Mitsui Sumitomo Insurance Welfare Foundation; Meiji Yasuda Life Foundation of Health and Welfare; Osaka Gas Group Welfare Foundation; Japan Diabetes Foundation; Daiwa Securities Health Foundation; Japan Science and Technology Agency; Takeda Science Foundation; YKK AP Inc.; Ushio Inc.; Nara Prefecture Health Promotion Foundation; Nara Medical University Grant-in-Aid for Collaborative Research Projects; Tokyo Electric Power Company; EnviroLife Research Institute Co., Ltd.; Sekisui Chemical Co., Ltd.; LIXIL Corp.; KYOCERA Corp.; ENDO Lighting Corp.; KANEKA Corp.; and Sompo Japan Nipponkoa Welfare Foundation.

Approval of the research protocol by an Institutional Reviewer Board: All procedures performed in studies involving human participants were in accordance with the ethical standards of the institutional and with the 1964 Helsinki declaration and its later amendments or comparable ethical standards. The protocol for this research project has been approved by a suitably constituted Ethics Committee of the institution and it conforms 
to the provisions of the Declaration of Helsinki. The ethics committee of Nara Medical University, Approval No. 301.

Informed consent: All informed consent was obtained from the subjects.

Conflicts of interest: Yoshiaki Tai received research grant from JSPS KAKENHI (grant number: 18K15899). Keigo Saeki and Kenji Obayashi received research grant from Mitsui Sumitomo Insurance Welfare Foundation, Japanese Diabetes Foundation, Osaka Gas Group Welfare Foundation, Japan Science and Technology Agency, YKK AP Inc., Ushio Inc., Nara Prefecture Health Promotion Foundation, Nara Medical University Grantin-Aid for Collaborative Research Projects, Tokyo Electric Power Company, EnviroLife Research Institute Co., Ltd., Sekisui Chemical Co., Ltd., LIXIL Corp., KYOCERA Corp., ENDO Lighting Corp., and KANEKA Corp. Kenji Obayashi received research grant from JSPS KAKENHI (grant number: 24790774, 15H04777, and 19H03903), Takeda Science Foundation, and Daiwa Securities Health Foundation. Keigo Saeki received research grant from JSPS KAKENHI (grant number: 22790567, 25860447, 25461393, 16K09478, and 20K10476). Yuki Yamagami received research grant from JSPS KAKENHI (grant number: 18H06396, 19K21475, and 21K17310), Mitsui Sumitomo Insurance Welfare Foundation, Meiji Yasuda Life Foundation of Health and Welfare, Osaka Gas Group Welfare Foundation, and Sompo Japan Nipponkoa Welfare Foundation. Norio Kurumatani received research grant from JSPS KAKENHI (grant number: 15H04777). All other authors have nothing to disclose.

\section{SUPPLEMENTARY MATERIAL}

Supplementary data related to this article can be found at https:// doi.org/10.2188/jea.JE20210471.

\section{REFERENCES}

1. Hashim H, Blanker MH, Drake MJ, et al. International Continence Society (ICS) report on the terminology for nocturia and nocturnal lower urinary tract function. Neurourol Urodyn. 2019;38:499-508.

2. Tikkinen KA, Johnson TM 2nd, Tammela TL, et al. Nocturia frequency, bother, and quality of life: how often is too often? A population-based study in Finland. Eur Urol. 2010;57:488-496.

3. Bosch JL, Weiss JP. The prevalence and causes of nocturia. J Urol. 2013;189:S86-S92.

4. Rahman SN, Cao DJ, Monaghan TF, et al. Phenotyping the association between nocturia and hypertension: a systematic review and meta-analysis. J Urol. 2021;205:1577-1583.

5. Tikkinen KA, Auvinen A, Johnson TM 2nd, et al. A systematic evaluation of factors associated with nocturia-the population-based FINNO study. Am J Epidemiol. 2009;170:361-368.

6. Pesonen JS, Cartwright R, Vernooij RWM, et al. The impact of nocturia on mortality: a systematic review and meta-analysis. J Urol. 2020;203:486-495.

7. Imamura T, Ishizuka O, Nishizawa O. Cold stress induces lower urinary tract symptoms. Int J Urol. 2013;20:661-669.

8. Yoshimura K, Kamoto T, Tsukamoto T, Oshiro K, Kinukawa N, Ogawa O. Seasonal alterations in nocturia and other storage symptoms in three Japanese communities. Urology. 2007;69:864870 .

9. Saeki K, Obayashi K, Kurumatani N. Indoor cold exposure and nocturia: a cross-sectional analysis of the HEIJO-KYO study. BJU Int. 2016;117:829-835.

10. Chapman CL, Benati JM, Johnson BD, Vargas NT, Lema PC, Schlader ZJ. Renal and segmental artery hemodynamics during whole body passive heating and cooling recovery. J Appl Physiol (1985). 2019;127:974-983.

11. Tai Y, Obayashi K, Yamagami Y, et al. Hot water bathing before bedtime and shorter sleep onset latency is accompanied by a higher distal-proximal skin temperature gradient in older adults. J Clin Sleep Med. 2021;17:1257-1266.

12. Tai Y, Saeki K, Yamagami Y, et al. Association between timing of hot water bathing before bedtime and night-/sleep-time blood pressure and dipping in the elderly: a longitudinal analysis for repeated measurements in home settings. Chronobiol Int. 2019;36:1714-1722.

13. Haghayegh S, Khoshnevis S, Smolensky MH, Diller KR, Castriotta RJ. Before-bedtime passive body heating by warm shower or bath to improve sleep: a systematic review and meta-analysis. Sleep Med Rev. 2019;46:124-135.

14. Kohara K, Tabara Y, Ochi M, et al. Habitual hot water bathing protects cardiovascular function in middle-aged to elderly Japanese subjects. Sci Rep. 2018;8:8687.

15. Kobayashi T SM, Iguchi K, Shimizu M, et al. Effect of evening foot bathing on older adults with night nocturia for night urination status and sleep status. Bull Fac Nurs Yamanashi Prefec Univ. 2014;16:1-9.

16. Kyoda Y, Okada M, Kato R, et al. Does cognitive behavioral therapy using a self-check sheet improve night-time frequency in patients with nocturia? Results of a multicenter randomized controlled trial. Int J Urol. 2021;28:444-449.

17. Obayashi K, Saeki K, Iwamoto J, et al. Positive effect of daylight exposure on nocturnal urinary melatonin excretion in the elderly: a cross-sectional analysis of the HEIJO-KYO study. J Clin Endocrinol Metab. 2012;97:4166-4173.

18. Matsuo S, Imai E, Horio M, et al; Collaborators developing the Japanese equation for estimated GFR. Revised equations for estimated GFR from serum creatinine in Japan. Am J Kidney Dis. 2009;53:982-992.

19. Craig CL, Marshall AL, Sjöström M, et al. International physical activity questionnaire: 12-country reliability and validity. Med Sci Sports Exerc. 2003;35:1381-1395.

20. Murase N, Katsumura T, Ueda C, et al. Validity and reliability of Japanese version of International Physical Activity Questionnaire. J Health Welfare Stat. 2002;49:1-9.

21. Burke WJ, Roccaforte WH, Wengel SP. The short form of the Geriatric Depression Scale: a comparison with the 30-item form. J Geriatr Psychiatry Neurol. 1991;4:173-178.

22. Imamura $T$, Ishizuka $\mathrm{O}$, Sudha GS, et al. A galenical produced from Ba-Wei-Die-Huang-Wan (THC-002) provides resistance to the cold stress-induced detrusor overactivity in conscious rats. Neurourol Urodyn. 2013;32:486-492.

23. Peier AM, Moqrich A, Hergarden AC, et al. A TRP channel that senses cold stimuli and menthol. Cell. 2002;108:705-715.

24. Shimizu N, Sugimoto K, Nozawa M, et al. Efficacy of ramelteon in patients with insomnia and nocturia. Low Urin Tract Symptoms. 2013;5:69-74.

25. Tei C, Horikiri Y, Park JC, et al. Acute hemodynamic improvement by thermal vasodilation in congestive heart failure. Circulation. 1995;91:2582-2590.

26. Janssen CW, Lowry CA, Mehl MR, et al. Whole-body hyperthermia for the treatment of major depressive disorder: a randomized clinical trial. JAMA Psychiatry. 2016;73:789-795.

27. Iwase S, Kawahara Y, Nishimura N, et al. A comparison of head-out mist bathing, with or without facial fanning, with head-out half-body low-water level bathing in humans - a pilot study. Int J Biometeorol. 2014;58:999-1005.

28. Negoro H, Kanematsu A, Yoshimura K, Ogawa O. Chronobiology of micturition: putative role of the circadian clock. J Urol. 2013; 190:843-849.

29. Buhr ED, Yoo SH, Takahashi JS. Temperature as a universal resetting cue for mammalian circadian oscillators. Science. 2010;330: 379-385.

30. Johnson TM 2nd, Burrows PK, Kusek JW, et al; Medical Therapy of Prostatic Symptoms Research Group. The effect of doxazosin, finasteride and combination therapy on nocturia in men with benign prostatic hyperplasia. J Urol. 2007;178:2045-2050. 
Tai Y, et al.

31. Oelke M, Roehrborn CG, D’Ancona C, Wilson TH, Castro R, Manyak M. Impact of dutasteride on nocturia in men with lower urinary tract symptoms suggestive of benign prostatic hyperplasia (LUTS/BPH): a pooled analysis of three phase III studies. World $J$ Urol. 2014;32:1141-1147.

32. Buser N, Ivic S, Kessler TM, et al. Efficacy and adverse events of antimuscarinics for treating overactive bladder: network metaanalyses. Eur Urol. 2012;62:1040-1060.
33. Japanese Ministry of Health, Labour and Welfare. 2010 National Health and Nutrition Survey. https://www.mhlw.go.jp/bunya/ kenkou/eiyou/h22-houkoku.html. Accessed August 1, 2021.

34. Obayashi K, Saeki K, Kurumatani N. Association between melatonin secretion and nocturia in elderly individuals: a crosssectional study of the HEIJO-KYO cohort. J Urol. 2014;191:18161821. 